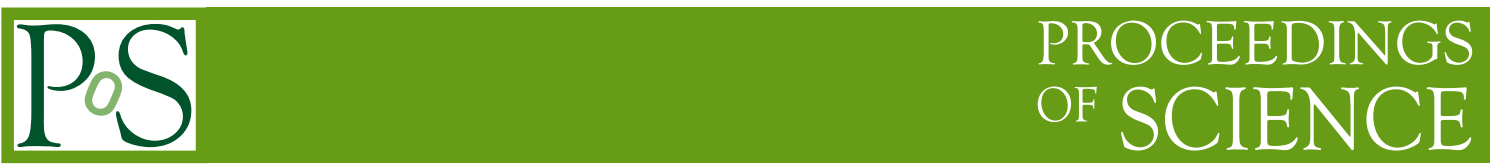

\title{
News on the CLIC physics potential
}

\author{
Aleksander Filip Żarnecki* ${ }^{*}$ on behalf of the CLICdp Collaboration \\ Faculty of Physics, University of Warsaw \\ E-mail: Filip.Zarnecki@fuw.edu.pl
}

The Compact Linear Collider (CLIC) is a proposed TeV-scale high-luminosity electron-positron collider. For an optimal exploitation of its physics potential, CLIC is foreseen to be built and operated in three stages, with centre-of-mass energies ranging from $380 \mathrm{GeV}$ up to $3 \mathrm{TeV}$. Electron beam polarisation is provided at all energies. The initial energy stage will focus on precision measurements of Higgs-boson and top-quark properties. The subsequent energy stages enhance the reach of many direct and indirect searches for new physics Beyond the Standard Model (BSM) and give access to the Higgs self-coupling. Higgs and top-quark projections have been evaluated using full detector simulation studies. Many new phenomenology studies have been undertaken to explore the BSM reach of CLIC, from Effective Field Theory (EFT) interpretations of precision measurements through to signature-based searches; these include flavour dynamics, and dark matter and heavy neutrino searches. Selected results that demonstrate the outstanding potential of CLIC in many physics domains are reviewed.

ALPS 2019 An Alpine LHC Physics Summit

April 22 - 27, 2019

Obergurgl, Austria

${ }^{*}$ Speaker. 


\section{Introduction}

The Compact Linear Collider (CLIC) is a mature option for a future electron-positron collider operating at centre-of-mass energies of up to $3 \mathrm{TeV}$. The conceptual design for a machine based on a two-beam acceleration scheme, allowing for accelerating gradients up to $100 \mathrm{MV} / \mathrm{m}$, was presented in 2012 [1]. For full exploitation of its physics potential CLIC will be built and operated in three stages [2], with accelerator footprint of 11 to $50 \mathrm{~km}$. The energy of $380 \mathrm{GeV}$ was chosen for the initial stage to optimize the physics potential in terms of Higgs-boson and top-quark measurements. Assuming 8 years of running at this stage, a total of $1 \mathrm{ab}^{-1}$ of data should be collected, including $100 \mathrm{fb}^{-1}$ at the $t \overline{\mathrm{t}}$ threshold. This is similar to the luminosity expected per interaction point for FCC-ee [3] with half the construction costs and half the power consumption of the initial stage of CLIC. Two high energy stages of CLIC, at $\sqrt{s}=1.5 \mathrm{TeV}$ with an assumed integrated luminosity of $2.5 \mathrm{ab}^{-1}$ and at $\sqrt{s}=3 \mathrm{TeV}$ with $5 \mathrm{ab}^{-1}$, will mainly focus on direct and indirect Beyond the Standard Model (BSM) searches, but additional Higgs boson and top-quark studies are also possible. Some of the rare Standard Model processes can only be measured at the TeV energies. CLIC is the only $\mathrm{e}^{+} \mathrm{e}^{-}$collider project that can go into this domain.

A dedicated CLIC detector concept, CLICdet, has been developed to assure full exploitation of physics potential from $380 \mathrm{GeV}$ to $3 \mathrm{TeV}$, based on detailed simulation studies, detector R\&D programme and beam tests [4]. The design is optimised for Particle Flow reconstruction [5] with expected jet energy resolution $\sigma_{E} / E=3-4 \%$ for high jet energies. High resolution tracking based on all-silicon vertex and tracker detectors will also allow for very efficient flavour tagging.

Presented in the contribution are selected results based on detector-level simulations from recent studies of the physics potential of CLIC. For more information on the CLIC project and its physics potential, please refer to the documents submitted as input to the European Strategy for Particle Physics Update 2018-2020 [6].

\section{Higgs physics}

Two main channels contribute to the Higgs boson production at CLIC: the Higgsstrahlung process, $\mathrm{e}^{+} \mathrm{e}^{-} \rightarrow \mathrm{ZH}$, and the WW-fusion process, $\mathrm{e}^{+} \mathrm{e}^{-} \rightarrow \mathrm{H} v \bar{v}$. Recoil mass reconstruction in Higgsstrahlung events allows for unbiased event selection, which is crucial for model-independent determination of the Higgs couplings [7]. The clean environment allows for unambiguous separation of different decay channels opening prospects for direct measurement of $\mathrm{BR}(\mathrm{H} \rightarrow \mathrm{c} \overline{\mathrm{c}})$ and setting stringent limits on the invisible Higgs boson decays. By combining all cross section measurements for the two production channels, the Higgs boson couplings can be extracted with a model-independent fit. For example, the coupling to the $\mathrm{Z}$ boson can be established with a precision of $0.6 \%$ at the first energy stage alone [8]. When including data collected at $1.5 \mathrm{TeV}$ and $3 \mathrm{TeV}$, most of the Higgs couplings can be measured at the percent level and the total width of the boson can be determined to 2.5\%. In Fig. 1 (left) CLIC sensitivity to the different Higgs boson couplings is compared with the HL-LHC projections, for a model-dependent analysis [9]. For some of the couplings, CLIC measurements will reduce their uncertainties by an order of magnitude. High energy stages of CLIC allow also the Higgs-boson pair production process to be studied. The trilinear Higgs self-coupling, which is crucial for understanding of electroweak symmetry breaking, can 

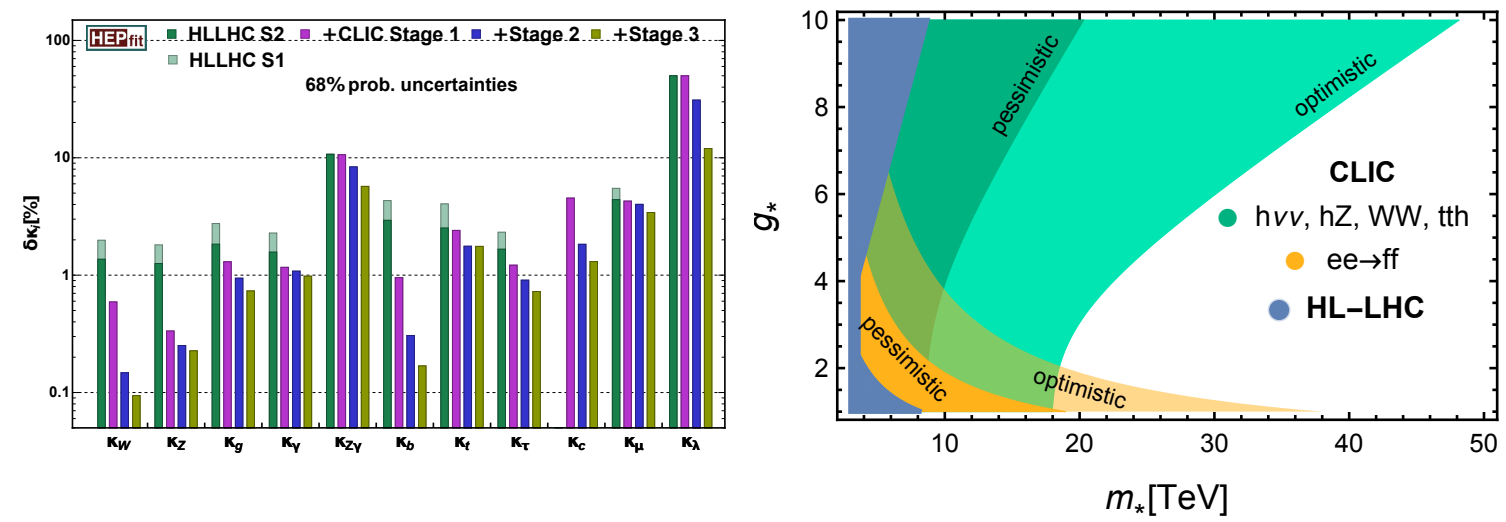

Figure 1: Left: CLIC sensitivity to the different Higgs boson couplings from the model-dependent fit (combined with the HL-LHC projections). Right: discovery $(5 \sigma)$ reach on Composite Higgs at CLIC, compared with expected exclusion limits $(2 \sigma)$ from the HL-LHC. Figures taken from [9].

be extracted from the measured kinematic distributions with uncertainty of the order of $10 \%$ [10]. Precision measurements at CLIC can be also used to search for or constrain different new physics scenarios. Shown in Fig. 1 (right) is the $5 \sigma$ discovery range for Higgs compositeness at CLIC compared to expected HL-LHC $2 \sigma$ exclusions limits [9]. For this type of model, the discovery reach of CLIC can extend beyond the HL-LHC energy scale.

\section{Top quark physics}

Already at the initial stage of CLIC over a million top quarks and anti-quarks will be produced. This will allow for detailed study of top-quark properties including top-quark mass and electroweak couplings, and searching for rare top-quark decays. For a direct mass measurement from reconstruction of top-quark hadronic decays a statistical precision of $30 \mathrm{MeV}$ is expected. However, the measurement requires excellent control of the jet energy scale and is subject to substantial theoretical uncertainties. The most precise determination of the top-quark mass, both in terms of the statistical and of the systematic uncertainties, is possible with a dedicated scan of the top pair production threshold. With $100 \mathrm{fb}^{-1}$ collected in 10 steps around the threshold (see Fig. 2 left), a statistical mass uncertainty of $20-30 \mathrm{MeV}$ is expected and a total systematic uncertainty of the order of $50 \mathrm{MeV}$ [11].

With large statistics of top quarks and anti-quarks produced, rare top-quark decays can be searched for. Expected limits for Flavour-Changing Neutral Current (FCNC) decays involving the $\mathrm{c}$ quark, assuming $1000 \mathrm{fb}^{-1}$ collected at $380 \mathrm{GeV}$, range from $2.6 \times 10^{-5}$ for $\mathrm{BR}(\mathrm{t} \rightarrow \mathrm{c} \gamma)$ to $3.4 \times 10^{-4}$ for a decay involving an invisible dark matter particle, $\mathrm{BR}(\mathrm{t} \rightarrow \mathrm{cE})$. FCNC top couplings can also be constrained from the measurement of single top production. These constraints are comparable to the direct ones for $380 \mathrm{GeV}$, but improve significantly for higher energy running [9].

The top-quark Yukawa coupling can be indirectly constrained from the threshold scan. However, the measurement is very sensitive to systematic effects. Direct determination of the top-quark Yukawa coupling is possible from the measurement of the tit $\mathrm{H}$ production cross section at high energy stages of CLIC. Signal event selection, with four b-jets expected in the final state, profits 

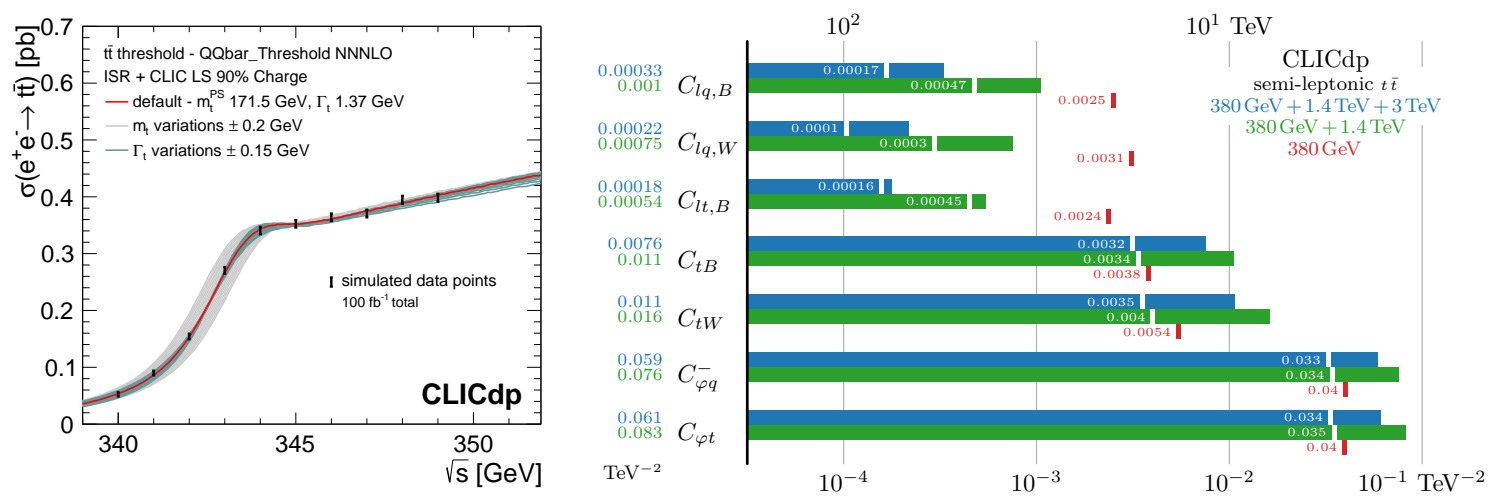

Figure 2: Left: top-quark mass determination from the dedicated threshold scan at CLIC. Right: summary of the global EFT analysis results using statistically optimal observables for the three CLIC energy stages. Figures taken from [11].

from the excellent flavour tagging capabilities. The expected precision on the top Yukawa coupling for $2.5 \mathrm{ab}^{-1}$ of data collected at $1.5 \mathrm{TeV}$ is $2.7 \%$.

Precise measurements of the top-quark pair-production cross-sections, forward-backward asymmetries and angle distributions in top decays can be use to set constraints on possible BSM contributions in the framework of Effective Field Theory (EFT) operators. Measurements at one energy stage are insufficient to simultaneously constrain all relevant EFT couplings. Only by combining data collected at different energies (and polarisations) all Wilson coefficients contributing to the top-quark pair production can be constrained simultaneously. Summary of the global EFT analysis results, based on statistically optimal observables, is shown in Fig. 2 (right). With high precision of top measurements CLIC is sensitive to "new physics" in the top sector up to scales in the $100 \mathrm{TeV}$ range.

\section{Beyond Standard Model physics}

While strong limits on mass scales of "new physics" are expected at HL-LHC for scenarios involving strongly interacting new particles, complementary searches are possible at CLIC. Both direct searches, for models with weak couplings or soft signatures, as well as high sensitivity indirect searches, based on precision measurements, were considered [9]. Sensitivity to possible "new physics" processes at large energy scales can be studied in the general framework of the EFT operators. By combining precision measurements of different Higgs and top-quark observables, as well as WW and two-fermion production processes, limits on the considered EFT operators can be set in the global analysis. As shown in Fig. 3, CLIC measurements will significantly improve limits expected from HL-LHC.

For many models, in particular those with an exotic scalar sector or new Higgs bosons, the CLIC direct and indirect reach can exceed that of HL-LHC. As an example, indirect and direct sensitivities to new heavy scalar singlets are shown in Fig. 4 (left). For the direct search, the heavy scalar decay to a pair of Higgs bosons, $\Phi \rightarrow \mathrm{HH} \rightarrow \mathrm{b} \bar{b} b \bar{b}$, was considered.

The Higgsino is one of the common targets in searches for supersymmetric extensions of the Standard Model. If it is not the lightest supersymmetric particle, the Higgsino can decay into a 


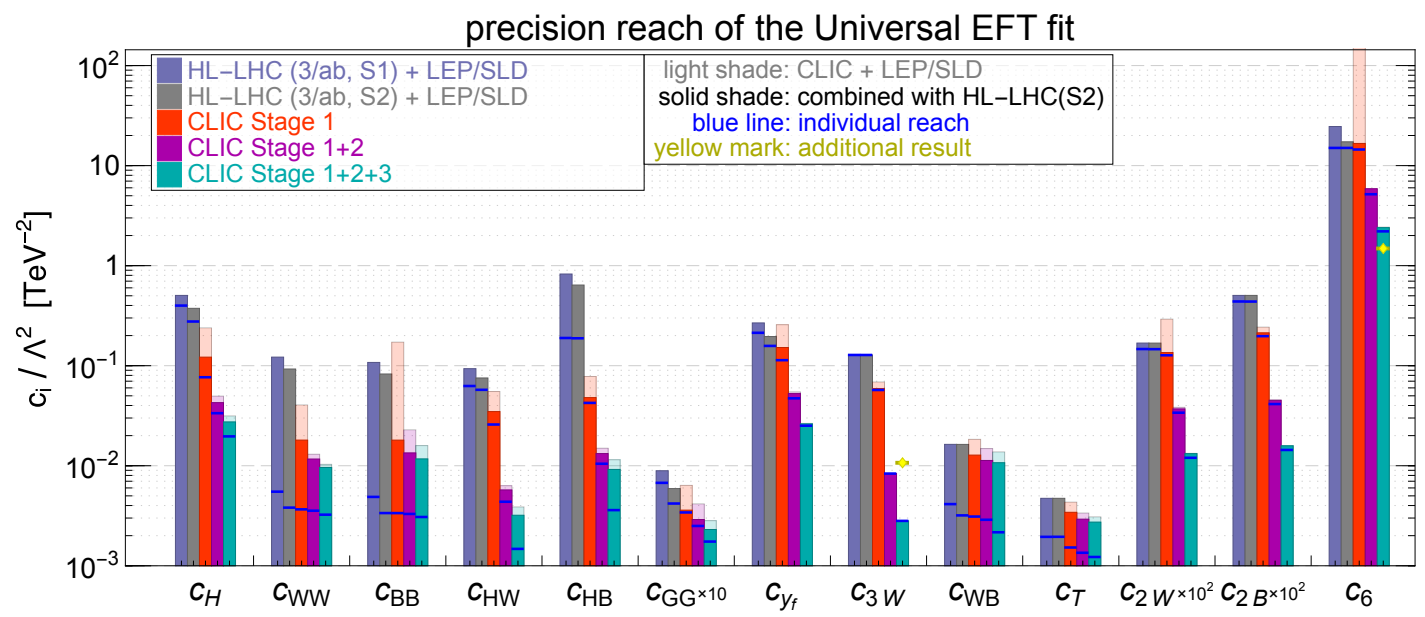

Figure 3: Summary of the CLIC sensitivity to EFT operators $c_{i} / \Lambda^{2}$ from a global analysis of Higgs and topquark observables, WW production, and two-fermion scattering processes, for three energy stages. Figure taken from [9].
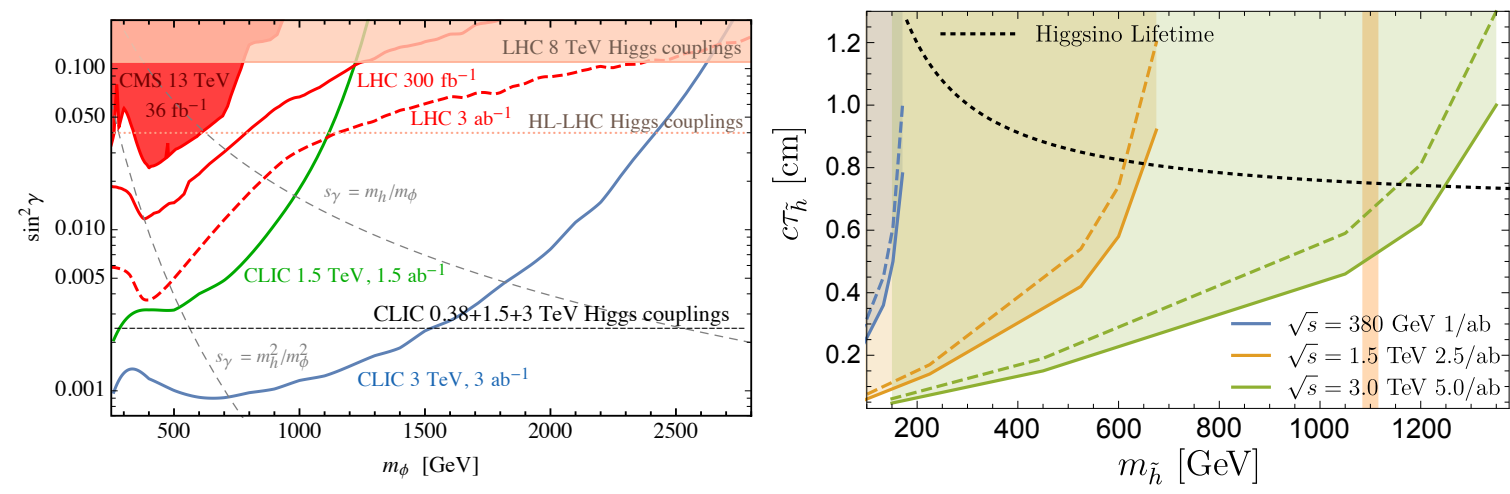

Figure 4: Left: expected 95\% C.L. exclusion limits from direct search for heavy scalar singlet production at CLIC compared with the expected limits from LHC and HL-LHC and the expected indirect limits from Higgs boson coupling measurements. Right: 95\% C.L. exclusion contours in lifetime-mass for $\mathrm{N}=3$ (solid) and $\mathrm{N}=30$ (dashed) Higgsino events in the detector acceptance at the three stages of CLIC. Figures taken from [2].

SM boson and missing energy. One of the possible signatures are the "disappearing tracks" caused by a heavy charged particle passing through the detector before decaying to a neutral particle that escapes detection and a very soft charged particle, unresolved in the detector. Search for dark matter production using "disappearing tracks" signature at CLIC profit from precision tracking and low background conditions. Expected exclusion contours for the three stages of CLIC are compared in Fig. 4 (right).

\section{Conclusions}

CLIC is an attractive and cost-effective option for a next large facility at CERN offering the unique combination of high collision energies and the clean environment of electron-positron collisions. The energy chosen for the initial CLIC stage is optimal for Higgs and top-quark measure- 
ments, allowing for precise determination of the top-quark mass and Higgs couplings, but also setting stringent constraints on many BSM scenarios. Subsequent CLIC stages reaching TeV scales will significantly enhance precision of SM measurements, and extend indirect BSM searches to $\mathscr{O}(100) \mathrm{TeV}$ scales and direct searches for "new physics" complementary to those planned at HLLHC up to the TeV scales.

\section{Acknowledgements}

This contribution was supported by the National Science Centre, Poland, the OPUS project under contract UMO-2017/25/B/ST2/00496 (2018-2021).

\section{References}

[1] M. Aicheler et al., A Multi-TeV Linear Collider Based on CLIC Technology : CLIC Conceptual Design Report, CERN-2012-007.

[2] P. N. Burrows et al. (CLICdp and CLIC Collaborations), The Compact Linear Collider (CLIC) - 2018 Summary Report, CERN-2018-005-M, arXiv:1812.06018.

[3] A. Abada et al. (FCC Collaboration), FCC-ee: The Lepton Collider : Future Circular Collider Conceptual Design Report Volume 2, Eur. Phys. J. ST 228 (2019) no.2, 261.

[4] D. Arominski et al. (CLICdp Collaboration), A detector for CLIC: main parameters and performance, CLICdp-Note-2018-005, arXiv:1812.07337.

[5] M. A. Thomson, Particle Flow Calorimetry and the PandoraPFA Algorithm, Nucl. Instrum. Meth. A 611 (2009) 25 [arXiv:0907.3577].

[6] CLIC input to the European Strategy for Particle Physics Update 2018-2020 https://clic.cern/european-strategy

[7] H. Abramowicz et al., (CLICdp Collaboration), Higgs physics at the CLIC electron-positron linear collider, Eur. Phys. J. C 77 (2017) no.7, 475, [arXiv:1608.07538].

[8] A. Robson and P. Roloff, Updated CLIC luminosity staging baseline and Higgs coupling prospects, CLICdp-Note-2018-002, arXiv:1812.01644.

[9] J. de Blas et al., The CLIC Potential for New Physics, CERN-2018-009-M, arXiv:1812.02093.

[10] P. Roloff et al., Double Higgs boson production and Higgs self-coupling extraction at CLIC, CLICdp-Note-2018-006, arXiv:1901.05897.

[11] H. Abramowicz et al. (CLICdp Collaboration), Top-Quark Physics at the CLIC Electron-Positron Linear Collider, CLICdp-Pub-2018-003, arXiv:1807.02441. 